\title{
Robust Modelling of Biological Neuroregulators
}

\author{
D. Ruiz Fernández, J.M. García Chamizo, F. Maciá Pérez, A. Soriano Payá
}

\begin{abstract}
The modelling of biological neuroregulated systems is usually complex because of their non-structured and distributed architecture. Software agents are a very useful tool to use in the modelling of this kind of systems, allowing increasing the model with characteristics which are not present in the biological equivalent. This work proposes a model of a neural regulator incorporating modularity, flexibility and scalability. The main approach has been the modelling of each neural centre as an agent, incorporating the distributed behaviour of the system. On the other hand, the model can solve local dysfunctions in the centres using extra diagnostic information. The neural regulator of the lower urinary tract has been implemented as an example. We have developed several experiments adding artificial dysfunctions to the model and comparing the results with a normal functioning of the model.
\end{abstract}

\section{INTRODUCTION}

$\mathrm{T}_{\mathrm{d}}^{\mathrm{H}}$ HE modelling of neuronal regulators is usually developed using theories with an important mathematical base [1], [2]. This type of modelling is difficult because most of the information about the behaviour is qualitative, a lot of data are incomplete and, furthermore, sometimes those systems present an emergent behaviour.

Most of the published models about neuronal regulators focus the problem from a global perspective. In this work we deal with the problem from a distributed point of view with emergent characteristics. It is in this context where the software agents approach offers a greater level of abstraction reducing the complexity of the problem [3]. This approach provides a framework capable of supplying reasonable expressive capacity to tackle the development of such distributed systems, accounting for a wide range of contingencies, emerging behaviour, and the possibility of structure modification as new advances are being made in neurological research.

In the following sections, the formal model based in the

Manuscript received April 30, 2005.

D. Ruiz Fernández is with the Department of Technology and Information Computing, University of Alicante, P.O. 99, 03080 Alicante, Spain (corresponding author to provide phone: +34 965903400 Ext 3331; fax: (+34) 965909643; e-mail: druiz@dtic.ua.es).

J.M. García Chamizo is with the Department of Technology and Information Computing, University of Alicante, P.O. 99, 03080 Alicante, Spain (e-mail: juanma@dtic.ua.es).

F. Maciá Pérez is with the Department of Technology and Information Computing, University of Alicante, P.O. 99, 03080 Alicante, Spain (e-mail: pmacia@dtic.ua.es).

A. Soriano Payá is with the Department of Technology and Information Computing, University of Alicante, P.O. 99, 03080 Alicante, Spain (e-mail: soriano@dtic.ua.es) software agents approach is presented; we explain properties like self-diagnostic and self-correction added to the model, which improve its robustness; then, experimentation with the neuronal regulator of the human lower urinary tract is shown; finally, we present the conclusions and the future lines of work.

\section{FORMAL MODEL}

At general level, we assume that a biological system is made up of a mechanical system (MS), a neuronal regulator system (NRS) that controls the mechanical part and an interface $\left({ }^{\mathrm{MS}} \mathrm{I}_{\mathrm{NRS}}\right)$ communicating both systems. Formally,

$$
\text { Biological_System }=\left\langle\mathrm{MS}, \mathrm{NRS},{ }^{\mathrm{MS}} \mathrm{I}_{\mathrm{NRS}}\right\rangle
$$

The interface regards the biological system as a system of actions and reactions, using the following structure:

$$
{ }^{\mathrm{MS}} \mathrm{I}_{\mathrm{NRS}}=\langle\Sigma, \Gamma, \mathrm{P}\rangle
$$

where $\Sigma$ represents the group of possible states of the system. $\Gamma$ identifies the group made up of the possible intentions of actions on the system. The agents do not have overall control of the system and they also have to combine their objectives with those of other agents so that the result of each action will be represented as an intention of action on the system. Finally, P is the set of all the possible actions that the entities can perform on the system.

The states of the system $(\Sigma)$ can be expressed by the values of the different sensor and actuator signals that act as an interface with the others systems. Each state $\sigma_{i} \in \Sigma$ is defined as a list of pairs (signal, valueSignal):

$$
\sigma_{\mathrm{i}}=\left\langle\left(\operatorname{sig}_{1}, \operatorname{val}_{1}\right),\left(\operatorname{sig}_{2}, \operatorname{val}_{2}\right), \ldots,\left(\operatorname{sig}_{\mathrm{u}}, \mathrm{val}_{\mathrm{u}}\right)\right\rangle
$$

On the other hand, the set of the possible influences or attempts of action of the different agents in reference to the present state from the system is defined as:

$$
\Gamma=\left\{\gamma_{1}, \gamma_{2}, \ldots, \gamma_{n}\right\}
$$

in which $\gamma_{i}$ is a list of pairs of an element together with its value.

The agents must carry out actions to be able to act on the system. The set of all possible actions that can be performed in a certain system can be defined as:

$$
\mathrm{P}=\left\{\mathrm{p}_{1}, \mathrm{p}_{2}, \ldots, \mathrm{p}_{\mathrm{n}}\right\}
$$

Each action $\left(\mathrm{p}_{\mathrm{i}}\right)$ is defined in terms of a name, a precondition that describes the conditions that must verify the action to be executed, and a post condition that makes 
inferences on the set of influences that converge on the action being executed.

The entities of the neuronal regulator system (NRS) are modelled as cognitive agents which are perceiving, deliberating and executing continually [3], [4]. The capacity to memorize has been incorporated to those agents in order to obtain a richer and more powerful deliberation.

An agent $\alpha \in$ NRS can be formally described using the structure:

$$
\alpha=\left\langle\Phi_{\alpha}, \mathrm{S}_{\alpha}, \text { Percept }_{\alpha}, \mathrm{Mem}_{\alpha}, \text { Decision }_{\alpha}, \text { Exec }_{\alpha}\right\rangle
$$

where $\Phi_{\alpha}$ corresponds to the set of perceptions; $\mathrm{S}_{\alpha}$ is the set of internal status; Percept $t_{\alpha}$ provides information about the state of the system; $\operatorname{Mem}_{\alpha}$ allows the centre to show awareness of the state; Decision $_{\alpha}$ selects the next influence; $\operatorname{Exec}_{\alpha}$ represents the agent's intention of acting on the system [5].

For an agent, perception is the quality of being able to classify and to distinguish states of the system. The perception is defined as a function that associates a set of values, denominated perceptions or stimuli, with a set of states of the system:

$$
\text { Percept }_{\alpha}: \Sigma \rightarrow \Phi_{\alpha}
$$

The set of the possible perceptions associated with the agent is defined as:

$$
\Phi_{\alpha}=\left\langle\phi_{1}, \phi_{2}, \ldots, \phi_{\mathrm{m}}\right\rangle
$$

where each $\phi_{\mathrm{i}}$ is a structure composed of a list of pairs formed by an element and its value corresponding to the state of the system previously defined.

Each agent has an internal state that confers the capacity to memorize and to develop a complex behaviour. The set of internal states of a certain agent is defined as:

$$
\mathrm{S}_{\alpha}=\left\{\mathrm{s}_{1}, \mathrm{~s}_{2}, \ldots, \mathrm{s}_{\mathrm{p}}\right\rangle
$$

On the other hand, the decision function submits an action to the perception in a determined internal state of the agent:

$$
\text { Decision }_{\alpha}: \Phi_{\alpha} \times \mathrm{S}_{\alpha} \rightarrow \mathrm{P}
$$

The decision function depends on the precondition decision function $\left(\operatorname{PreD}_{\alpha}(\phi, s)\right)$ that relates a true or false value to a perception in a given internal state, a function $\left(\operatorname{FunD}_{\alpha}(\phi, s)\right)$ associates a list of actuators signals the new values the agent has acquired a perception function.

The memorization function of information happens when switching to another internal state; that is, it will relate an internal state of the agent to a perception in a given internal state:

$$
\mathrm{Mem}_{\alpha}: \Phi_{\alpha} \times \mathrm{S}_{\alpha} \rightarrow \mathrm{S}_{\alpha}
$$

The memorization function depends on a precondition memorization function $\left(\operatorname{PreM}_{\alpha}(\phi, s)\right)$ that relates a true or false value to a perception in a particular internal state, a function $\left(\operatorname{FunD}_{\alpha}(\phi, s)\right)$ that associates a new internal state with a perception in a given internal state.

Once the agent has decided what action to take, it must execute. The actions on the system are carried out by means of the execution function defined as

$$
\operatorname{Exec}_{\alpha}: \mathrm{P} \times \Phi_{\alpha} \rightarrow \Gamma
$$

This paper focuses on the neuronal regulator of a biological system. However, since the mechanical system (MS) and the neuronal regulator system are so closely connected, let's introduce the function of the mechanical system: to generate afferent signals from a given set of efferent signals [6]. This function complies with the dynamics of the mechanical system and is carried out through accomplishment of actions with the aim of transforming one state into another. This change is regarded as reaction of the system under different influences.

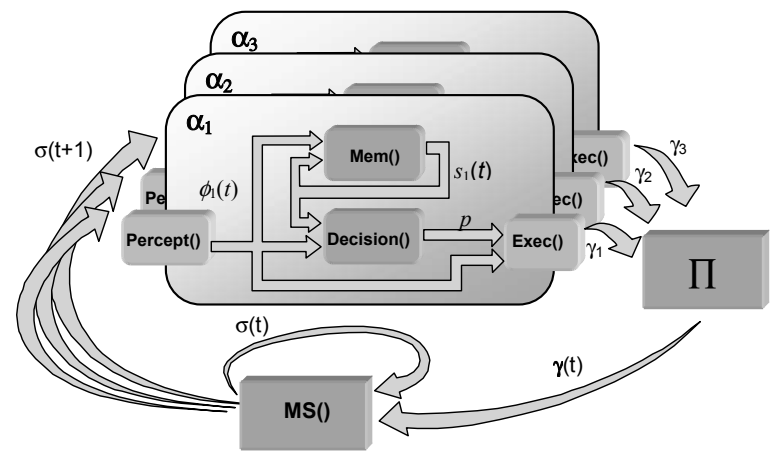

Fig. 1. Multiagent system. The internal structure of the agents and the relation with the mechanical system is shown.

Function MS() provides the information about the current state of the system subjected to the influences from different entities (fig. 1):

$$
\text { MS : } \Sigma \times \Gamma \rightarrow \Sigma
$$

Taking into account the above definition for agent, and the perception of the environment at a certain point, the new state of the system results as the assessment of influences from the different agents when they are concurrently performing their tasks:

$\sigma(\mathrm{t}+1)=\operatorname{MS}\left(\sigma(\mathrm{t}), \prod_{\mathrm{i}}^{\mathrm{v}} \operatorname{Exec}_{\mathrm{i}}\left(\operatorname{Decision}_{\mathrm{i}}\left(\phi_{\mathrm{i}}(\mathrm{t}), \mathbf{s}_{\mathrm{i}}(\mathrm{t})\right), \phi_{\mathrm{i}}(\mathrm{t})\right)\right) ;$

$\mathbf{s}_{\mathbf{1}}(\mathrm{t}+1)=\operatorname{Mem}_{\mathrm{i}}\left(\phi_{1}(\mathrm{t}), \mathbf{s}_{\mathbf{1}}(\mathrm{t})\right) ; \ldots ; \mathbf{s}_{\mathbf{v}}(\mathrm{t}+1)=\operatorname{Mem}_{\mathrm{n}}\left(\phi_{\mathbf{v}}(\mathrm{t}), \mathbf{s}_{\mathbf{v}}(\mathrm{t})\right)$

with:

$$
\phi_{\mathrm{i}}(\mathrm{t})=\operatorname{Percept}_{\mathrm{i}}(\sigma(\mathrm{t}))
$$

The model presented can incorporate qualitative information related with the behaviour of each agent thanks to the specification of the set of states and the internal functions. The concurrent execution of the agents can show a global emergent behaviour.

\section{ROBUSTNESS}

The model presented reproduces not only the functioning of the neuronal regulator but also the distributed structure. 
The level of similarity makes that the model inherit the problems of the biological system. One of the most important problems is the risk that a dysfunction in one of the centres that participate in the regulation could affect to the general functioning of the system.

To avoid the previous problem is necessary to identify a dysfunction when it happens in a neuronal centre and to apply a mechanism to correct it.

In order to keep the structure and flexibility of the system, we will include the knowledge of diagnostic as near as possible to its origin, so that the information about the correct states (corresponding to a normal behaviour) of a neuronal centre will be included in its own structure [7]. We add an evaluation process in the deliberation function: after the agent decides which one is the following state, it is
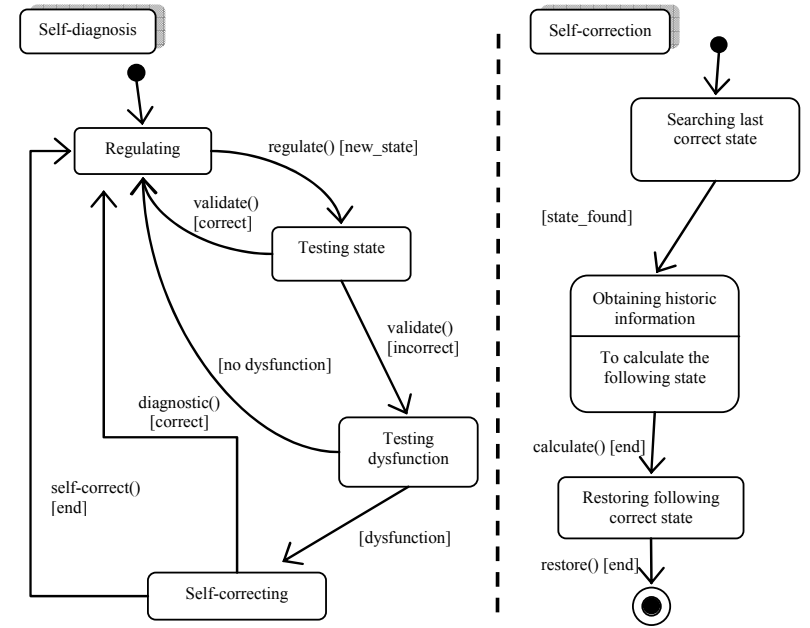

Fig. 2. State diagram of the self-diagnostic and self-correction characteristics.

tested if the future state is coherent with the dynamics of functioning of the agent. If the result of the test is negative, then there is a dysfunction and it is necessary to apply a solution (fig 2).

To implement the activity of self-correction it is possible to use the historical behaviour of each centre. The memory of an agent will store several sequences of states of functioning. The dynamics of an agent $\alpha$ can be represented as a history $h$ that is made up of a sequence of internal states:

$$
h^{\alpha}=\left(s_{0}^{\alpha}, s_{1}^{\alpha}, \ldots, s_{m}^{\alpha}\right)
$$

We define a history-window $\left(V_{h}\right)$ as the quantity of states which are stored in a history $h$. The typical behaviour of an agent is defined by the set of all histories during the life of the agent. We consider that an agent has a dysfunction when an incorrect state is repeated inside a history (dysfunctional history). On the contrary, the correct histories are those which have only correct states:

$$
h_{\alpha}=\left(s_{1}, \ldots, s_{n}\right) / n=V_{h_{\alpha}}, s_{i} \in S_{\alpha}^{C} \quad \forall i=1 \ldots n
$$

with $\mathrm{S}_{\alpha}{ }^{\mathrm{C}}$ the set of correct states that the agent $\alpha$ can get.

The historical information of functioning will be used to solve a dysfunction following an idea of continuity: the behaviour of an agent is repetitive (the same than the system regulated) and, sometimes, cyclic (as many human biological functions). Because of this it is possible to know the estimated behaviour of the agent according to its historical evolution. When a dysfunction is detected, the agent applies the last correct history that continues the cycle in the moment of the dysfunction.

Sometimes abnormal states can appear sporadically during a normal situation so it is important to be in mind that the apparition of an incorrect state does not imply a dysfunction. It is necessary that the incorrect activity (the same incorrect state or another) continues during a period of time.

\section{EXPERIMENTS}

In order to study the model proposed we have implemented the system of the neuronal regulator of the lower urinary tract (LUT).

The lower urinary tract carries out two main functions: storage of urine in the bladder and the expulsion of urine via the urethra (micturition process) [8], [9]. This process mainly depends of the coordination between the detrusor muscle and the external sphincter. The LUT can be divided in two parts: the mechanical system (MSLUT) and the neuronal regulator (RLUT). The first system describes the biomechanics of the LUT and it is related to the anatomy and physiology of the muscles and tissues which belongs to the LUT system. The second one is related to the neuronal control pathways and the activating and inhibiting centres associated to the micturition.

We can find three types of communication in the system:

-- Afferent signals, from the mechanical system to the neuronal centres with information about the state

-- Efferent signals, from the neuronal centres to the mechanical system, which acts on the muscles allowing their contraction or relaxation.

-- Internal signals, information of control between the neuronal centres.

On the ground of the formal framework as proposed in section 2, we formally define the lower urinary tract (LUT) using:

$$
\text { LUT }=\left\langle\text { MLUT, RLUT, }{ }^{\text {MLUT }} \mathrm{I}_{\text {RLUT }}\right\rangle
$$

where the MLUT models the mechanical system of the lower urinary tract, the RLUT, the neuronal regulator of the lower urinary tract, and finally, the ${ }^{\mathrm{MLUT}} \mathrm{I}_{\mathrm{RLUT}}$ correlates them.

The neuronal regulator of the lower urinary tract consists of a group of nine neuronal centres that are constantly perceiving, deliberating and executing. 


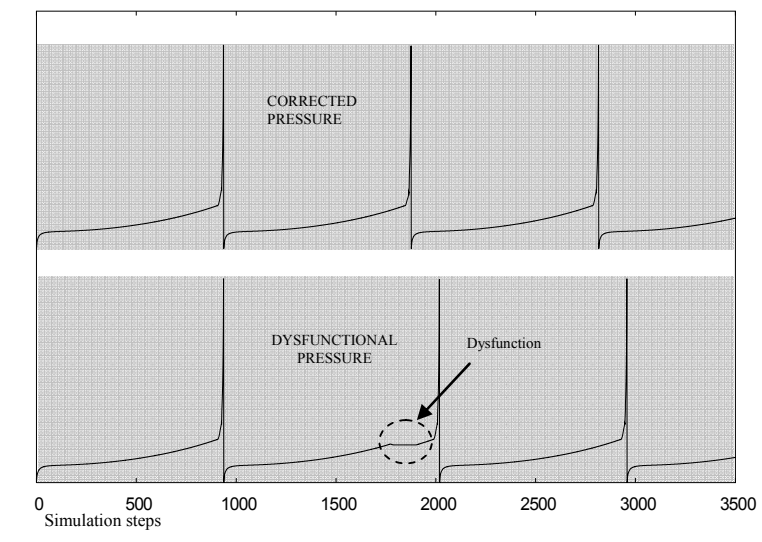

Fig. 3. Graphs of pressure in the bladder in a normal situation and other in which a dysfunction has been simulated.

The states of system $\left(\sigma_{i}\right)$ are a list of pairs consisting of the afferent and efferent neuronal signals with their corresponding values. The influences of a neuronal centre $\left(\gamma_{i}\right)$ are stated by a list of pairs of its efferent neuronal signals together with the new values that the neuronal centre wants to obtain.

In the experimentation that has been carry out we have tested the robustness of the model inserting some functioning mistakes in the agents, representing dysfunctions of the neuronal centres, and studying the resulting behaviour of the model. We have compared this behaviour with the one obtained with the model without dysfunctions.

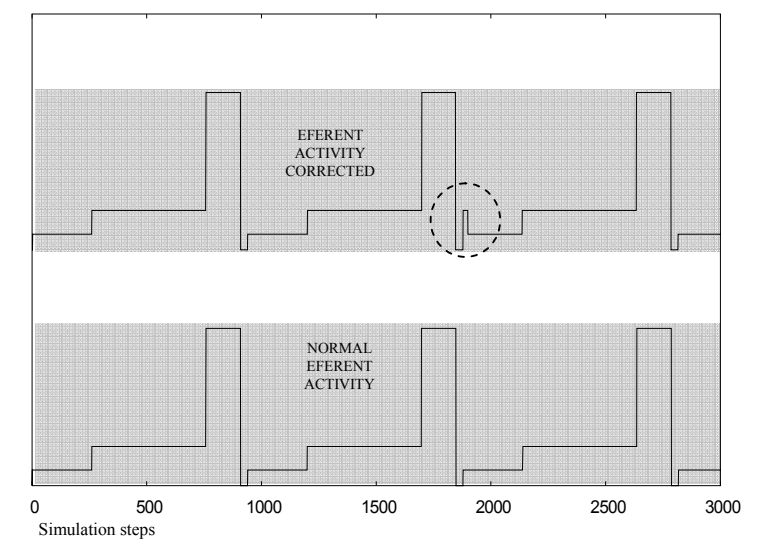

Fig. 4. Graphs of efferent activity in a normal situation and in a corrected dysfunctional situation.

In the test we show we have introduced a dysfunction in the sacral storage (SS) centre that is related with the storage phase. We have modified the efferent signals of the agent $\left({ }^{\mathrm{SS}} \mathrm{E}_{\mathrm{EUS}}\right.$ and ${ }^{\mathrm{SS}} \mathrm{E}_{\mathrm{PF}}$ ) to the external urethral sphincter and to the pelvic floor in order to get a global dysfunction that provokes a relaxation of the muscles and leaks of urine. In the figure 3 we can observe this situation: the stabilization of pressure means a dysfunction. It is also possible to see that the behaviour of the model with characteristics of selfdiagnostic and correction is completely normal; there is not any stabilization of the pressure and the peaks of pressure are the same than in a situation without dysfunctions.

In the figure 4 it is shown the efferent activity in a normal situation versus a dysfunctional situation that has been selfcorrected. We can observe in the graphs corresponding to the corrected activity that there is a small abnormal step which is the indication of the beginning of a dysfunction. The situation is solved quickly and the emergent behaviour of the system is completely normal as we can see in the figure 4.

\section{CONCLUSION}

In this paper, it has been presented a model of neuronal regulators with characteristics of robustness thanks to properties added as self-diagnostic and self-correction.

The agents paradigm gives enough flexibility to incorporate in the model the necessary knowledge for the monitoring of functioning and the implementation of the correction task.

The robustness of the model has been tested using an implementation of the neuronal regulator of the lower urinary tract. The graphs of urodynamical measures obtained in situations with dysfunctions were almost the same that in normal behaviours and in all cases the values were in normal ranges.

The robustness presented in the model allows increasing the reliability of external controllers that collaborate with biological neuronal regulators.

As main future lines of research we think to experiment the model in other areas, biological or not, and to study the use of the model as simulator to learn and practice in the studies of urology.

\section{REFERENCES}

[1] K.J. Aström (ed.): Control of Complex Systems. Spinger-Verlag 2000.

[2] K. Ogata, Modern Control Engineering,.Prentice Hall, $3^{\mathrm{a}}$ ed., 1998.

[3] J. Ferber, Multi-Agent Systems. An Introduction to Distributed Artificial Intelligence. Addison-Wesley.1999.

[4] A. Soriano Payá, "Modeling and simulation of the neuronal regulador of the lower urinary tract", Ph.D. dissertation, Dept. Comp. Tech, University of Alicante, Alicante, Spain, 2001.

[5] F. Maciá Pérez, "Modeling of administration of heterogeneous of computer networks. System of regeneration of nodes of networks". Ph.D. dissertation, Dept. Comp Tech, University of Alicante, Alicante, Spain, 2001.

[6] D. Ruiz Fernández, "Modeling of self-regulation of biological systems. Characterization and correction of neurogen urinary dysfunctions in human", Ph.D. dissertation, University of Alicante, Alicante, Spain, 2003.

[7] D. Ruiz Fernández, J.M. García Chamizo, F. Maciá Pérez, A. Soriano Payá, "Modeling the Distributed Control of the Lower Urinary Tract Using a Multiagent System", Lecture Notes in Artificial Intelligence, Springer-Verlag 3131 (2004) 104 -114.

[8] B.F.M. Blok, G. Holstege, "The central control of micturition and continence: implications for urology". British Journal of Urology, 83 (1999) 1-6.

[9] M.V. Kinder, E.H.C. Bastiaanssen, R.A. Janknegt, E. Marani, "The Neuronal Control of the Lower Urinary Tract: A Model of Architecture and Control Mechanisms". Archives of Physiology and Biochemistry, 107 (1999) 203-222. 